\title{
Diagnostic Accuracy of MRI-Based Morphometric Parameters for Detecting Olfactory Nerve Dysfunction
}

\author{
(D)M.K. Lee, (D).H. Lee, (D).H. Kim, (D)H. Kim, (D)L. Joo, (D). Kim, (D).J. Cho, (D).H. Suh,
} (D) S.R. Chung, (D).J. Choi, and DJ.H. Baek

\begin{abstract}
BACKGROUND AND PURPOSE: Although olfactory dysfunction is a common cranial nerve disorder, there are no simple objective morphometric criteria to assess olfactory dysfunction. The aim of this study was to evaluate the diagnostic performance of MR imaging morphometric parameters for detecting olfactory dysfunction.
\end{abstract}

MATERIALS AND METHODS: This prospective study enrolled patients from those presenting with olfactory symptoms who underwent both an olfactory function test and MR imaging. Controls without olfactory dysfunction were recruited during the preoperative work-up for pituitary adenoma. Two independent neuroradiologists measured the olfactory bulb in 3D and assessed olfactory bulb concavity on MR imaging while blinded to the clinical data. Diagnostic performance was assessed using receiver operating characteristic curve analysis.

RESULTS: Sixty-four patients and 34 controls were enrolled. The patients were significantly older than the controls (mean age, $57.8 \pm 11.9$ years versus $47.1 \pm 12.1$ years; $P<.001$ ). Before age adjustment, the olfactory bulb height was the only olfactory bulb parameter showing a significant difference between patients and controls $(1.6 \pm 0.3 \mathrm{~mm}$ versus $2.0 \pm 0.3 \mathrm{~mm}, P<.001)$. After age adjustment, all parameters and olfactory bulb concavity showed significant intergroup differences, with the olfactory bulb height having the highest area under the curve (0.85). Olfactory bulb height was confirmed to be the only significant parameter showing a difference in the detection of olfactory dysfunction in 22 pairs after matching for age and sex (area under the curve $=0.87$, $P<.001)$. Intraclass correlation coefficients revealed moderate-to-excellent degrees of inter- and intrareader agreement.

CONCLUSIONS: MR imaging morphometric analysis can differentiate patients with olfactory dysfunction, with the olfactory bulb height having the highest diagnostic performance for detecting olfactory dysfunction irrespective of age.

ABBREVIATIONS: APD = anterior-posterior diameter; $\mathrm{AUC}=$ area under the curve

O lfactory dysfunction is a common cranial nerve disorder. In population-based studies, the prevalence of olfactory dysfunction is about $19 \%-24 \%$, with this rate being higher in older men. ${ }^{1-3}$ The etiologies of olfactory loss include postviral upper respiratory infection, sinonasal disease, head trauma, aging, congenital causes, toxins/drugs, idiopathic loss, and neurologic disorders

Received April 29, 2020; accepted after revision June 9.

From the Department of Radiology and Research Institute of Radiology (M.K.L., J.H.L., H.K., L.J., M.K., S.J.C., C.H.S., S.R.C., Y.J.C., J.H.B.), and Department of Otorhinolaryngology (J.H.K.), University of Ulsan College of Medicine, Asan Medical Center, Seoul, Republic of Korea; and Department of Radiology (M.K.L.), Yeouido St. Mary's Hospital, College of Medicine, The Catholic University of Korea, Seoul, Korea.

Please address correspondence to Jeong Hyun Lee, MD, PhD, Department of Radiology and Research Institute of Radiology, University of Ulsan College of Medicine, Asan Medical Center, 86 Asanbyeongwon-Gil, Songpa-Gu, Seoul 05505, Republic of Korea; e-mail: jeonghlee@amc.seoul.kr

Indicates article with supplemental on-line table.

http://dx.doi.org/10.3174/ajnr.A6697 such as Parkinson disease and Alzheimer disease. ${ }^{4}$ Olfaction serves as a warning for hazards such as poisonous fumes and microbial harm and helps with food intake. ${ }^{5}$ Loss of olfaction can result in severe problems, including loss of personal hygiene and social communication and reduction in the quality of life. ${ }^{6}$

Precise assessment of a patient's olfactory status should be made ahead of starting treatment, to predict the prognosis and provide proper counseling. Olfactory function is generally assessed by using olfactory function tests such as the Sniffin' Sticks test (Burghardt), ${ }^{7}$ the University of Pennsylvania Smell Identification Test, ${ }^{8}$ and the Connecticut Chemosensory Clinical Research Center Threshold test. ${ }^{9}$ MR imaging can provide anatomic information on the olfactory pathway, and the evaluation of olfactory dysfunction using imaging parameters has been attempted previously. ${ }^{4,10,11}$ The volume of the olfactory bulb is one such imaging parameter, with changes in volume being known to correlate with olfactory loss or odor-threshold 


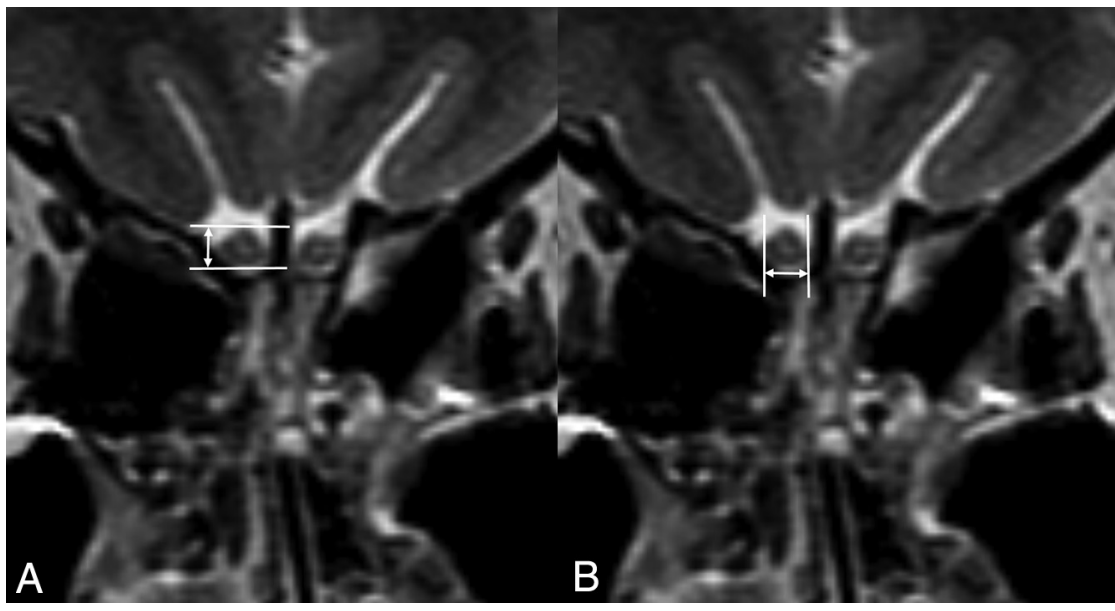

FIG 1. A 41-year-old male control subject. Coronal T2WI is used to show how to measure the height $(A)$ and width $(B)$ of the olfactory bulb, 2.8 and $3.5 \mathrm{~mm}$, respectively.

changes. ${ }^{12-14}$ However, measurement of olfactory bulb volume is not commonly used to evaluate the olfactory pathway in routine practice because volumetric analysis using planimetric manual contouring of multiple sections is difficult and complicated compared with visual analysis. ${ }^{11,13-15}$ Recently, Chung et al ${ }^{4}$ suggested that olfactory bulb atrophy seen on MR imaging can be used to objectively detect olfactory dysfunction in patients with subjective olfactory loss. Despite the suppleness of their method, their report was limited by the use of a subjective definition of olfactory bulb atrophy, and corrections were not made for patient age.

To make MR imaging diagnosis more robust, repeatable, and reproducible, we are still in need of simple objective morphometric criteria to assess olfactory dysfunction. Furthermore, although olfactory function is known to be affected by aging, ${ }^{2}$ no published study has evaluated the effects of aging on olfactory bulb size. Therefore, the purpose of our study was to evaluate simple morphometric parameters of the olfactory bulb measured on MR imaging and to determine their diagnostic accuracy in patients with subjective olfactory dysfunction, making comparisons with healthy controls after age adjustment and matching.

\section{MATERIALS AND METHODS Study Population}

Our institutional review boards Asan Medical Center approved this prospective study, and written informed consent was obtained from all participants. The study enrolled patients from those who presented with subjective olfactory nerve symptoms at a single tertiary referral center between March 2017 and November 2018. The enrollment criterion was olfactory nerve dysfunction revealed by the threshold-discrimination-identification score. ${ }^{16}$ According to previous studies using the Korean version of the Sniffin' Sticks test II kit, ${ }^{4,16,17}$ those patients with a threshold-discrimination-identification score of $<28$ were diagnosed as having objective olfactory nerve dysfunction. The enrolled patients agreed to undergo MR imaging with protocols dedicated to imaging the olfactory nerve.

The control participants were recruited during preoperative work-up for functioning pituitary adenoma. The subjects were routinely screened by a questionnaire for olfactory dysfunction, which was ruled out using the threshold-discrimination-identification score for subjective olfactory dysfunction. MR imaging of the olfactory nerve was added to the preoperative imaging of the pituitary mass after obtaining informed consent. The exclusion criteria were an age younger than 18 years, history of a previous endoscopic transnasal or endonasal operation, history of skull base fracture, radiation treatment to the head and neck area, active sinonasal disease, and psychiatric or neurologic conditions.

The presumptive causes of olfactory dysfunction were classified into 4 categories: post-upper respiratory infection, ${ }^{4,14}$ chronic rhinosinusitis, ${ }^{18}$ posttraumatic or postsurgical causes, ${ }^{4,14}$ and idiopathic causes. Idiopathic olfactory dysfunction was defined when patients did not have a definite cause for the subjective olfactory nerve dysfunction after an extensive evaluation. ${ }^{19}$ The patients' medical histories were evaluated, especially for the presence of Parkinson disease because olfactory dysfunction is a common symptom in Parkinson disease, having a similar frequency to resting tremor. ${ }^{20-22}$

\section{MR Imaging Acquisition}

MR images were acquired on a 3T unit (Ingenia 3T CX; Philips Healthcare) with a 64-channel head and neck coil. The imaging sequences used to evaluate the olfactory nerve included coronal 2D-T2WI. The imaging parameters for the 2D-T2WI included the following: matrix, $512 \times 512$; TR/TE, $300 / 80 \mathrm{~ms}$; FOV, $90 \times$ $190 \mathrm{~mm}$; section thickness, $1.5 \mathrm{~mm}$ without interval; voxel size, $0.43 \times 0.43 \times 1.5 \mathrm{~mm}$; bandwidth, $209 \mathrm{~Hz} /$ pixel; TSE factor, 15 ; scan time, 3 minutes.

\section{MR Imaging Analysis}

Two independent board-certified neuroradiologists with 22 and 6 years of experience assessed the MR images and measured the following morphometric parameters on 2D T2WI: olfactory bulb height and width and anterior-posterior diameter (APD) of the olfactory bulb (Fig 1). The olfactory bulb height and width were measured directly on the largest cross-sectional image of the olfactory bulb using coronal T2WI. The APD was measured by summation of the slices containing a visible olfactory bulb. The neuroradiologists also assessed the presence of olfactory bulb concavity, defined as the line connecting both edges of the bulb being equal to or higher than the center of the bulb on coronal sections (Fig 2). The measurements were performed blinded to the patients' clinical information. To assess intrareader agreement, 1 radiologist repeated the analysis within a 2 -week interval.

\section{Statistical Analyses}

The means of the olfactory bulb height, width, and APD measurements made on both sides of a patient were used for 


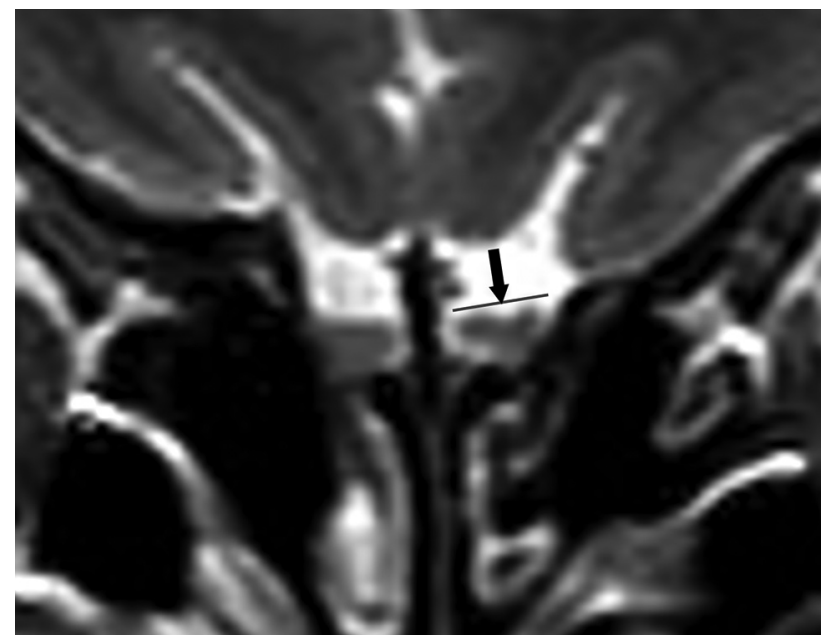

FIG 2. A 59-year-old female patient with idiopathic olfactory dysfunction with a threshold-discrimination-identification score of 14. Coronal T2WI shows olfactory bulb concavity (arrow) on the left side compared with the normal oval shape on the right side. The olfactory bulb heights and lateral diameters were 2.1 and $3.5 \mathrm{~mm}$, respectively, on the right side and 1.3 and $3.5 \mathrm{~mm}$ on the left side.

Table 1: Summary of the demographic data of the patient and control groups

\begin{tabular}{lccr}
\hline & $\begin{array}{c}\text { Patients } \\
(\boldsymbol{n}=64)\end{array}$ & $\begin{array}{c}\text { Controls } \\
(\boldsymbol{n}=\mathbf{3 4})\end{array}$ & $\begin{array}{c}\boldsymbol{P} \\
\text { Value }\end{array}$ \\
\hline${\text { Age }(\mathrm{yr})^{\mathrm{a}}}^{\mathrm{b}}$ & $57.8 \pm 11.9$ & $47.1 \pm 12.2$ & $<.001$ \\
Male sex $^{\text {Duration of symptoms (yr) }}$ & $29(45)$ & $14(41)$ & .910 \\
Threshold-discrimination-identification & $6.3 \pm 12.7$ & $\mathrm{NA}$ & \\
$\quad$ score & $15.7 \pm 6.7$ & $\mathrm{NA}$ & \\
$\begin{array}{l}\text { Presumptive cause of olfactory } \\
\quad \text { dysfunction }\end{array}$ & & & \\
$\begin{array}{l}\text { Chronic rhinosinusitis } \\
\text { Post-upper respiratory infection }\end{array}$ & $23(36)$ & NA & \\
Posttraumatic or postsurgical & $16(25)$ & NA & \\
Idiopathic & $7(11)$ & NA & \\
\hline
\end{tabular}

Note:-NA indicates not applicable.

${ }^{a}$ Numbers are presented as mean \pm SD.

${ }^{b}$ Number of patients. Numbers in parentheses are percentages.

Table 2: Morphometric parameters in the patient and control groups

\begin{tabular}{lccc}
\hline MR Imaging Parameters & Patients $(\boldsymbol{n}=64)$ & Controls $(\boldsymbol{n}=\mathbf{3 4})$ & $\boldsymbol{P}$ Value \\
\hline APD $(\mathrm{mm})^{\mathrm{a}}$ & $11.9 \pm 1.9$ & $12.5 \pm 1.8$ & .107 \\
${\text { Width }(\mathrm{mm})^{\mathrm{a}}}^{\mathrm{a}}$ & $4.1 \pm 0.5$ & $4.1 \pm 0.4$ & .881 \\
${\text { Height }(\mathrm{mm})^{\mathrm{a}}}_{\text {Olfactory bulb concavity }^{\mathrm{b}}}^{1.6 \pm 0.3}$ & $2.0 \pm 0.3$ & $<.001$ \\
\hline
\end{tabular}

${ }^{a}$ Numbers are presented as mean \pm SD.

${ }^{b}$ Number of patients. Numbers in parentheses are percentages.

the statistical analysis. The primary outcomes were the differences in the measured olfactory bulb height, width, APD, and olfactory bulb concavity between patients and controls. Demographic data are presented using descriptive statistics. Continuous variables were assessed for normal distribution using the Shapiro-Wilk test. Continuous data were analyzed with the Student $t$ test or Mann-Whitney test according to the results of the Shapiro-Wilk test. Nominal data were analyzed with the $\chi^{2}$ test.
Because there was a significant difference in age distribution between the patients and controls, a logistic regression model including age and each morphometric parameter as a covariate was developed to detect olfactory nerve dysfunction. The discriminative ability of this model was evaluated using receiver operating characteristic curve analysis and the area under the curve (AUC). The discriminative ability of the morphometric parameters was also evaluated in 22 pairs of patients and controls matched for age and sex to control for selection bias. The balance of the matches was checked using the standardized mean difference. Interreader and intrareader agreement were assessed using intraclass correlation coefficients. A $P$ value $<.05$ was considered statistically significant. Statistical analysis was performed using $\mathrm{R}$ statistical and computing software (R version 3.6.1; http://www. r-project.org/) and MedCalc (MedCalc Software).

\section{RESULTS}

Among the 103 patients who visited with subjective olfactory nerve symptoms during the study period, 64 patients (male/ female ratio $=29: 35$; mean age, 57.8 years; range, $22-84$ years) were finally enrolled and underwent olfactory nerve MR imaging after exclusion of 39 patients with normal results on the thresholddiscrimination-identification test. The presumptive causes for olfactory nerve dysfunction were chronic rhinosinusitis in 23 patients, post-upper respiratory infection in 16 , and posttraumatic or postsurgical causes in 7 , while 18 cases were idiopathic. Two patients had a history of Parkinson disease. The average duration of olfactory dysfunction was $6.3 \pm 12.7$ years.

Thirty-four subjects (male/female ratio $=14: 20$; mean age, 47.1 years; range, 26-69 years) were enrolled as controls. The patients were significantly older than the controls (mean age, $57.8 \pm 11.9$ versus $47.1 \pm$ 12.2 years; $P<.001$ ), but there was no significant difference in sex distribution (Table 1).

Table 2 shows the differences in morphometric parameters between patients and controls. The patients had significantly smaller olfactory bulb heights $(1.6 \pm 0.3 \mathrm{~mm}$ versus $2.0 \pm 0.3 \mathrm{~mm}, P<.001)$ than the controls (Fig 3). Olfactory bulb concavity was seen more frequently in patients than in controls, but the difference was not statistically significant ( $41 \%$ versus $24 \%, P=.13$ ). All the parameters demonstrated moderate-to-excellent interreader and intrareader agreement (intraclass correlation coefficient = 0.69-0.85; On-line Table 1).

On-line Table 2 shows the diagnostic performance of the morphometric parameters for differentiating patients from controls before and after age adjustment in all cases and in 22 pairs 

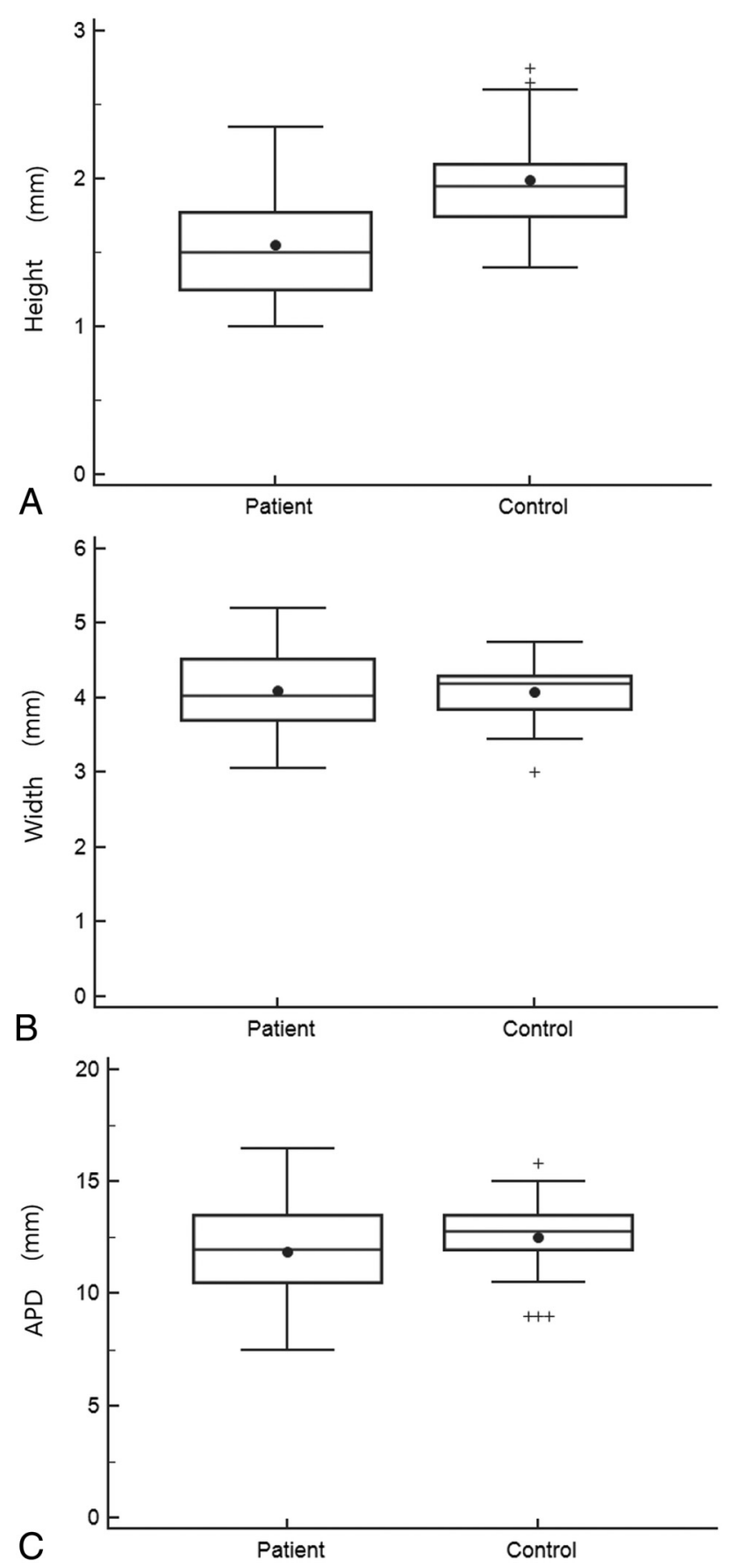

FIG 3. Box-and-whisker plots of the olfactory bulb height $(A)$, width $(B)$ and anterior-posterior diameters (C), showing comparisons between patients and controls. The solid circles represent the mean values of the parameters, and the horizontal lines represent the median values (median patient olfactory bulb height $=1.5 \mathrm{~mm}$, median control olfactory bulb height $=2.0 \mathrm{~mm}$; median patient olfactory bulb width $=4.0 \mathrm{~mm}$, median control olfactory bulb width $=4.2 \mathrm{~mm}$; median patient $A P D=12.0 \mathrm{~mm}$, median control APD $=12.8 \mathrm{~mm}$ ). The tops and bottoms of the boxes represent the upper and lower quartiles, the tops and bottoms of the whiskers represent the highest and lowest observations, and the crosses represent outliers.

after matching for age and sex. Before age adjustment, olfactory bulb height was the only parameter showing significant differences between patients and controls in the detection of olfactory dysfunction $(\mathrm{AUC}=0.83 ; 95 \% \mathrm{CI}, 0.75-0.91 ; P<.001)$. After age adjustment, all the morphometric parameters showed a significant difference between patients and controls (all, $P<.001$ ), with olfactory bulb height having the highest AUC value (AUC= 0.85; 95\% CI, 0.77-0.93; $P<.001)$. After matching for age and sex, olfactory bulb height remained the only parameter showing a significant difference in detecting olfactory dysfunction (AUC = 0.87; 95\% CI, 0.74-0.95; $P<.001$ ).

\section{DISCUSSION}

Our study revealed that a simple morphometric parameter of the olfactory bulb could be useful for detecting patients with olfactory nerve dysfunction. Given that age is a significant factor affecting the morphometry of the olfactory bulb and olfactory bulb height was the only parameter significantly different between patients and controls before and after matching, we suggest that olfactory bulb height is potentially the best single parameter to assess olfactory dysfunction on MR imaging.

There have been previous studies evaluating imaging features of olfactory nerve dysfunction, with volume measurement of the olfactory bulb by planimetric manual contouring on each section of an MR image sequence being one of the reported methods. ${ }^{10,11,13,15,23}$ The authors reported positive correlations between olfactory performance and olfactory bulb volume in patients with different presumptive causes, including post-upper respiratory infection, head trauma, idiopathic loss, and Parkinson disease. Some researchers also evaluated the depth of the olfactory sulcus but found no significant correlation with olfactory function in patients with idiopathic olfactory loss. ${ }^{13,15}$ Although the olfactory bulb volume typically shows a significant correlation with olfactory function, the method is difficult to apply in routine clinical practice.

Recently, Chung et $\mathrm{al}^{4}$ proposed relatively simple imaging criteria to diagnose olfactory dysfunction. They used visual assessment of the olfactory bulb on coronal T2WI, 3D-T2-FLAIR, and 3D-T2 volume isotropic TSE acquisition images and reported that MR imaging evaluation of olfactory bulb atrophy can be used to diagnose olfactory dysfunction in patients with subjective olfactory loss. They defined olfactory bulb atrophy as flattening and thinning of the olfactory bulb, with loss of the normal oval or J-shape ${ }^{24}$ and an asymmetric decrease in the size of the olfactory bulb compared with that on the contralateral side. ${ }^{25}$ However, despite the suppleness of the method, we still need quantitative and repeatable morphometric parameters to objectively detect olfactory dysfunction.

Our prospective study is important because of the following features: First, we propose a simple morphometric analytic tool to detect olfactory dysfunction with excellent interreader and intrareader agreement, which is easily and readily applicable in routine clinical practice using thin-section T2WI. Thin-section TSE T2WI has advantages over the steady-state sequences such as CISS or FIESTA, which are commonly used to evaluate the cranial nerves in the cisternal spaces. TSE T2WI is free from the banding artifacts of the steady-state sequences occurring in the areas of air/bone and soft-tissue interfaces such as the olfactory sulcus. Moreover, the intrinsic contrast resolution of a nerve is better in spin-echo images compared with the CISS sequence. ${ }^{26}$ Second, we found that age was not only a significant factor influencing olfactory performance but that it also showed a relationship with the size of the olfactory bulb on clinical MR imaging. In previous cross-sectional 
studies, ${ }^{2,27-29}$ olfactory function and pathophysiologic change showed significant relationships with the aging process, but no study has previously shown age-related change in the size of the olfactory bulb on MR imaging. This is the first study to demonstrate such a relationship. Finally, we provide a clearer definition of olfactory bulb concavity, one that is similar to the concept of olfactory atrophy described by Chung et al. ${ }^{4}$

Despite these merits, there are also several limitations to our study. First, although we demonstrated that our simple morphometric analysis could be useful for detecting olfactory dysfunction, we could not suggest the size criteria for defining olfactory dysfunction for any of the parameters except olfactory bulb concavity, partly due to the relatively small sample size. Further investigation to determine the optimal olfactory morphometry size criteria for diagnosing olfactory dysfunction should be performed on larger cohorts using subject groups with even age distributions. Second, we could not compare the morphometric parameters among patients with different presumptive causes of olfactory dysfunction. Third, the APD was calculated by summing up the section numbers showing the olfactory bulb, not by directly measuring the olfactory bulb on sagittal or axial images. Direct measurement of APD might be more helpful for diagnosing olfactory dysfunction; however, our method has the advantage of being quite simple and able to be performed on a single MR imaging sequence.

\section{CONCLUSIONS}

Our study demonstrated that morphometric parameters that can be simply measured on coronal T2WI are helpful for objectively assessing olfactory dysfunction in patients with subjective olfactory dysfunction, and it also showed that age is a significant factor affecting olfactory bulb size. Among the measured parameters, olfactory bulb height shows the best potential for detecting olfactory dysfunction irrespective of age. However, a further larger-scale study with an even age distribution between groups is necessary to suggest diagnostic cutoff values for the parameters.

Disclosures: Jung Hwan Baek—UNRELATED: Consultancy: radiofrequency, Comments: consultant of STARmed Healthcare and radiofrequency medical companies from 2017.

\section{REFERENCES}

1. Brämerson A, Johansson L, Ek L, et al. Prevalence of olfactory dysfunction: the Skövde population-based study. Laryngoscope 2004;114:73337 CrossRef Medline

2. Murphy C, Schubert CR, Cruickshanks KJ, et al. Prevalence of olfactory impairment in older adults. JAMA 2002;288:2307-12 CrossRef Medline

3. Vennemann MM, Hummel T, Berger K. The association between smoking and smell and taste impairment in the general population. J Neurol 2008;255:1121-26 CrossRef Medline

4. Chung MS, Choi WR, Jeong HY, et al. MR imaging-based evaluations of olfactory bulb atrophy in patients with olfactory dysfunction. AJNR Am J Neuroradiol 2018;39:532-37 CrossRef Medline

5. Stevenson RJ. An initial evaluation of the functions of human olfaction. Chem Senses 2010;35:3-20 CrossRef Medline

6. Croy I, Nordin S, Hummel T. Olfactory disorders and quality of life: an updated review. Chem Senses 2014;39:185-94 CrossRef Medline

7. Hummel T, Sekinger B, Wolf SR, et al. 'Sniffin' sticks': olfactory performance assessed by the combined testing of odor identification, odor discrimination and olfactory threshold. Chem Senses 1997;22:3952 CrossRef Medline

8. Doty RL, Shaman P, Kimmelman CP, et al. University of Pennsylvania Smell Identification Test: a rapid quantitative olfactory function test for the clinic. Laryngoscope 1984;94:176-78 CrossRef Medline

9. Cain WS, Gent JF, Goodspeed RB, et al. Evaluation of olfactory dysfunction in the Connecticut Chemosensory Clinical Research Center. Laryngoscope 1988;98:83-88 CrossRef Medline

10. Buschhuter D, Smitka M, Puschmann S, et al. Correlation between olfactory bulb volume and olfactory function. Neuroimage 2008;42:498502 CrossRef Medline

11. Haehner A, Rodewald A, Gerber JC, et al. Correlation of olfactory function with changes in the volume of the human olfactory bulb. Arch Otolaryngol Head Neck Surg 2008;134:621-24 CrossRef Medline

12. Mueller A, Rodewald A, Reden J, et al. Reduced olfactory bulb volume in post-traumatic and post-infectious olfactory dysfunction. Neuroreport 2005;16:475-78 CrossRef Medline

13. Rombaux $P$, Potier H, Markessis E, et al. Olfactory bulb volume and depth of olfactory sulcus in patients with idiopathic olfactory loss. Eur Arch Otorhinolaryngol. 2010;267:1551-56 CrossRef Medline

14. Rombaux P, Huart C, Deggouj N, et al. Prognostic value of olfactory bulb volume measurement for recovery in postinfectious and posttraumatic olfactory loss. Otolaryngol Head Neck Surg 2012;147:113641 CrossRef Medline

15. Wang J, You H, Liu JF, et al. Association of olfactory bulb volume and olfactory sulcus depth with olfactory function in patients with Parkinson disease. AJNR Am J Neuroradiol 2011;32:677-81 CrossRef Medline

16. Hong SC, Yoo YS, Kim ES, et al. Development of KVSS Test (Korean Version of Sniffin' Sticks Test). Korean J OtorhinolaryngolHead Neck Surg 1999;42:855-60

17. Cho JH, Jeong YS, Lee YJ, et al. The Korean version of the Sniffin' stick (KVSS) test and its validity in comparison with the cross-cultural smell identification test (CC-SIT). Auris Nasus Larynx 2009;36:280-86 CrossRef Medline

18. Fokkens WJ, Lund VJ, Mullol J, et al. EPOS 2012: European position paper on rhinosinusitis and nasal polyps 2012: a summary for otorhinolaryngologists. Rhinology 2012;50:1-12 CrossRef Medline

19. Hoekman PK, Houlton JJ, Seiden AM. The utility of magnetic resonance imaging in the diagnostic evaluation of idiopathic olfactory loss. Laryngoscope 2014;124:365-68 CrossRef Medline

20. Ansari K, Johnson A. Olfactory function in patients with Parkinson's disease. J Chronic Dis 1975;28:493-97 CrossRef Medline

21. Doty RL, Deems DA, Stellar S. Olfactory dysfunction in parkinsonism: a general deficit unrelated to neurologic signs, disease stage, or disease duration. Neurology 1988;38:1237-37 CrossRef Medline

22. Mesholam RI, Moberg PJ, Mahr RN, et al. Olfaction in neurodegenerative disease: a meta-analysis of olfactory functioning in Alzheimer's and Parkinson's diseases. Arch Neurol 1998;55:84-90 CrossRef Medline

23. Rombaux P, Mouraux A, Bertrand B, et al. Olfactory function and olfactory bulb volume in patients with postinfectious olfactory loss. Laryngoscope 2006;116:436-39 CrossRef Medline

24. Schneider JF, Floemer F. Maturation of the olfactory bulbs: MR imaging findings. AJNR Am J Neuroradiol 2009;30:1149-52 CrossRef Medline

25. Booth TN, Rollins NK. Spectrum of clinical and associated MR imaging findings in children with olfactory anomalies. AJNR Am J Neuroradiol 2016;37:1541-48 CrossRef Medline

26. Li Z, Chen YA, Chow D, et al. Practical applications of CISS MRI in spine imaging. Eur J Radiol Open 2019;6:231-42 CrossRef Medline

27. Doty RL, Shaman P, Applebaum SL, et al. Smell identification ability: changes with age. Science 1984;226:1441-43 CrossRef Medline

28. Doty RL, Kamath V. The influences of age on olfaction: a review. Front Psychol 2014;5:20 CrossRef Medline

29. Duffy VB, Backstrand JR, Ferris AM. Olfactory dysfunction and related nutritional risk in free-living, elderly women. J Am Diet Assoc 1995;95:879-84 CrossRef Medline 